\title{
Estrogen Treatment Effects on Anticholinergic-Induced Cognitive Dysfunction in Normal Postmenopausal Women
}

\author{
Julie Dumas', Catherine Hancur-Bucci', Magdalena Naylor', Cynthia Sites ${ }^{2}$ and Paul Newhouse ${ }^{*, 1}$ \\ 'Clinical Neuroscience Research Unit, Department of Psychiatry, University of Vermont College of Medicine, Burlington, VT, USA; ${ }^{2}$ Division of \\ Reproductive Endocrinology, Department of Obstetrics and Gynecology, University of Alabama School of Medicine, Birmingham, AL, USA
}

\begin{abstract}
Estrogen has been shown to interact with the cholinergic system and influence cognition in animal models. This study investigated the interaction of estrogen and cholinergic system functioning and the effects of this interaction on cognitive task performance in healthy older women. Fifteen post-menopausal women were randomly and blindly placed on I mg of 17- $\beta$ estradiol or placebo for 3 months after which they participated in five anticholinergic challenge sessions, where they were administered one of two doses of the antimuscarinic drug scopolamine (SCOP) or the antinicotinic drug mecamylamine (MECA) or placebo. After the first challenge phase, they were crossed over to the other hormone treatment for another 3 months and repeated the challenges. Performance in multiple domains of cognition was assessed during anticholinergic drug challenge, including attention and verbal and nonverbal learning and memory. Results showed that estrogen pretreatment attenuated the anticholinergic drug-induced impairments on tests of attention and tasks with speed components. This study is the first to demonstrate the interaction of estrogen and the cholinergic system and the effects on cognitive performance in humans. The results suggest that estrogen status may affect cholinergic system tone and may be important for cholinergic system integrity.

Neuropsychopharmacology (2006) 3 I, 2065-2078. doi: I 0. I 038/sj.npp. I 30 I042; published online I 5 February 2006
\end{abstract}

Keywords: estrogen; cholinergic system; scopolamine; mecamylamine; menopause; cognition

\section{INTRODUCTION}

The prophylactic and therapeutic use of gonadal steroids is among the most controversial areas in medicine. The complex impact of these agents on various body systems has become necessary to understand, in particular for aging women. While significant strides have been made in elucidating the actions of these molecules on target organs such as bone, breasts, and cardiovascular systems, relatively little is known about their short- or long-term impact on the brain and brain function in humans.

As a result of menopause and the decrease in circulating estrogen, women may experience changes in cognitive functioning. Estrogen treatment (ET) has been shown to improve or at least maintain current levels of cognitive functioning in postmenopausal women (ie, Duka et al, 2000; Resnick et al, 1997); however, there is contradictory evidence (ie, Shumaker et al, 2003, 2004). While many

\footnotetext{
*Correspondence: Dr P Newhouse, Clinical Neuroscience Research Unit, Department of Psychiatry, University of Vermont College of Medicine, I South Prospect Street, Burlington, VT 0540I, USA. Tel: + I 802847 4560, Fax: + I 8028477889 ,

E-mail: paul.newhouse@uvm.edu

Received 20 September 2005; revised 24 December 2005; accepted 28 December 2005

Online publication: 5 January 2006 at http://www.acnp.org/citations/ Npp0 I0506050583/default.pdf
}

studies in humans have investigated the effects of estrogen and hormone therapy on cognition, none have proposed a mechanism of action for estrogen in the brain. One possible site of action for estrogen in the brain is the cholinergic system. Studies in rodents and primates have suggested potent effects of estrogen on cholinergic function (Tinkler and Voytko, 2005). Further, the activity of the CNS cholinergic system may be a primary determinant of the effectiveness of attentional, learning, and memory mechanisms (Sarter et al, 2005).

The current study utilized a controlled experimental design to investigate the interaction of estrogen and the cholinergic system and the effects of this interaction on cognition. Below is a brief review of the prior findings of ET on cognition, the evidence for the interaction of estrogen and the cholinergic system, and a description of the current study's examination of the interaction of estrogen and the cholinergic system in postmenopausal women.

Cognitive symptoms reported by women at menopause include difficulties in memory, attention, and word-finding. Studies have shown an acceleration of cognitive problems of aging after menopause, including difficulties on tests of driving simulation, reaction time, and some visual spatial tasks (Halbreich et al, 1995). There is some evidence that ET results in the maintenance of a premenopausal level of cognitive functioning (ie, Duka et al, 2000; Jacobs et al, 1998; Resnick et al, 1997; Smith et al, 2001a; Zandi et al, 
2002), and may reduce the risk of Alzheimer's disease (AD; Paganini-Hill and Henderson, 1994; Panidis et al, 2001; Tang et al, 1996). Some of the most consistent evidence for a direct effect of estrogen on cognitive function is from studies of surgically menopausal women. Sherwin (1988) studied women after total abdominal hysterectomy (TAH/ $\mathrm{BSO}$ ) with random assignment to estrogen or placebo for 3 months and found that the treated grouped showed preservation of verbal memory, while the placebo group showed a significant decline. These data were confirmed in a study showing that women treated with estrogen for 3 months after TAH/BSO showed either improvement in or preservation of verbal learning and memory performance compared to placebo-treated women who showed a decline (Phillips and Sherwin, 1992). In a naturalistic study, women who were placed on estrogen after surgical menopause had better preserved verbal memory and constructional ability than women not placed on estrogen (Verghese et al, 2000).

While many studies have shown positive effects of ET on cognition, there are a number of studies that showed no benefit (Barrett-Connor and Kritz-Silverstein, 1993; Binder et al, 2001; Ditkoff et al, 1991; Polo-Kantola et al, 1998). Recent findings from the Women's Health Initiative (WHI) and Women's Health Initiative Memory Study (WHIMS) have suggested that gonadal steroids may increase the risk of dementia. The WHIMS study found that the risk of diagnosis of dementia in women taking estrogen alone and estrogen and progesterone was twice that of women in the placebo group (Shumaker et al, 2003, 2004). In addition, in women without dementia, no overall cognitive benefit was seen (Rapp et al, 2003). In another study also using estrogen and progesterone, no benefit for cognition was seen after 4 years of follow-up (Grady et al, 2002). However, the majority of the dementia diagnoses in the WHI study seemed to be related to vascular disease (Vermeer et al, 2003), and women were an average age of 68 when entering the study. These issues may limit the generalizeability of the WHI/WHIMS findings, especially regarding the role of estrogen and progesterone (separately or in combination) in the preservation of normal cognition functioning (Yaffe, 2003).

Thus, prospective and some longitudinal studies indicate beneficial effects of estrogen replacement on cognitive functioning of postmenopausal women. One possible site of action explaining estrogen's effects on cognition is through its interaction with the cholinergic system (Tinkler and Voytko, 2005). Estrogen has been shown to modulate cholinergic neurotransmission in the brain (Gibbs, 1996; McMillan et al, 1996). Estrogen is associated with increases in choline uptake and choline acetyltransferase (ChAT) in the hippocampus and frontal lobe (Simpkins et al, 1997). Estrogen administration also appears to alter hippocampal structure and function (Bi et al, 2001; Woolley and McEwen, 1993). Loss of estrogen after ovariectomy in animals has been shown to decrease high affinity choline uptake, ChAT activity, and ChAT mRNA levels (Gibbs et al, 1994; Luine et al, 1975, 1986; Singh et al, 1993). These effects can be reversed by exogenous estrogen (McMillan et al, 1996). Cholinergic fiber density in the dorsolateral prefrontal cortex decreased 2 years after ovariectomy relative to intact monkeys, and this decrease was prevented if monkeys were treated with estrogen (Tinkler et al, 2004). Another study showed that cholinergic fiber density was restored in ovariectomized monkeys only 1 month after estrogen treatment (Kritzer and Kohama, 1999). The finding that estrogen affects cholinergic circuits in the frontal lobe and hippocampus is important because these areas are associated with cognitive performance and may explain why some studies find that estrogen has beneficial effects on cognition.

A number of studies have investigated the interaction of estrogen and the cholinergic system and its effects on memory performance in animals. Packard (1998) found that the posttraining administration of estrogen enhances memory relative to vehicle-trained rats in a time dependent manner and was blocked by the muscarinic antagonist scopolamine (SCOP). Daniel and Dohanich (2001) found a comparable increase in NMDA receptor binding in the CA1 area of the hippocampus when estrogen and physostigmine, an acetylcholinesterase inhibitor, were given to female rats. When given in combination with estrogen, the amounts of cholinergic agonist and glutamatergic agonist needed to produce good retention of a foot shock response was decreased 10-fold (Farr et al, 2000). In addition, the detrimental effects of SCOP on memory for passive avoidance were attenuated when rats were given estrogen (Gibbs et al, 1998). Lesions of the cholinergic system appear to alter the ability of estrogen to modify attentional performance in rats (McGaughy and Sarter, 1999). Thus, there is experimental evidence for the attenuation of the detrimental effects of cholinergic antagonists on memory when estrogen is present.

In humans, the cholinergic system has been implicated in many aspects of cognition, including the partitioning of attentional resources, working memory, inhibition of irrelevant information, and improved performance on effort-demanding tasks (see Newhouse et al (2001) for a review). Beginning with studies by Drachman and colleagues (Drachman, 1977; Drachman and Leavitt, 1974), temporary blockade of muscarinic receptors produced impairments of learning and memory that resembled changes associated with normal aging (Ghoneim and Mewaldt, 1977; Peterson, 1977). This has been proposed as a model of the cognitive deficits in AD (Bartus et al, 1982; Caine et al, 1981; Sitaram et al, 1978). These studies were extended by Sunderland and Newhouse (Newhouse et al, 1988; Sunderland et al, 1985), who showed that when elderly normals and elderly depressed patients were given SCOP, their performance declined to a level similar to AD patients. $\mathrm{AD}$ patients were even more sensitive to the impairing effects of the drug (Sunderland et al, 1988).

Data from studies using nicotinic antagonists in humans also show the detrimental effects of these drugs on cognition. Newhouse et al $(1992,1993,1994)$ used the nicotinic antagonist mecamylamine (MECA) to examine cognitive functioning in younger adults, healthy older adults, and patients with mild to moderate AD. MECA impaired cognitive performance in all groups in an age- and disease-dependent way. Younger adults showed impairments only after the largest dose of MECA (Newhouse et al, 1992), older adults show impairments after the medium and largest doses (Newhouse et al, 1994), and AD patients showed impairments at all doses (Newhouse et al, 1993). The impairments were manifest in learning phases of the 
tasks, but not during retrieval. These findings implicate a role for nicotinic receptors in learning and storing new information, but not in retrieving old information.

Thus, normal cognitive performance is disrupted when subjects are administered antimuscarinic and antinicotinic drugs creating a temporary lesion. The current study uses this model to probe the ability of estrogen to modulate the temporary cognitive impairment during anticholinergic drug challenge. Thus far, no study in humans has directly probed estrogen's effects on specific neurotransmitterrelated cognitive performance, although several studies have examined estrogen effects on neurotransmitter availability (Gardiner et al, 2004; Smith et al, 2001b; van Amelsvoort et al, 2001). We predicted that impairments in cognitive performance during cholinergic challenge would be attenuated after 3 months of estrogen treatment. The effects of estrogen were predicted be largest on tasks where the estrogen-cholinergic system interaction was critical for accurate performance on those tasks, in particular tests of attention and verbal episodic memory.

\section{METHODS}

\section{Subjects}

Subjects were 15 cognitively normal women, ages 48-84, $M=60.4(S D=10.3)$. These women were postmenopausal for an average of 11.4 years since their last menses $(S D=13.55)$. They had a mean body mass index (BMI) of $24.30(S D=3.46)$. Subjects had an average of 15.64 years of education $(S D=2.47)$. They were recruited through notices and advertisements in local newspapers and direct mailings. Subjects were required to be postmenopausal, nonsmokers without menses for 1 year, without surgically induced menopause, with no history of breast cancer, and no use of hormone therapy for at least 1 year. Three subjects had previously taken hormone or ET after menopause. The length of time of prior hormone use ranged from 2 weeks to 1.5 years. Medical exclusion criteria for ET included: contraindications for hormone therapy, estrogen-dependent neoplasia, untreated blood pressure greater than 160/100, history of deep vein thrombosis or other thromboembolic disease, hepatoma, severe migraines or stroke on oral contraceptives, current use of barbiturates, rifampin, insulin, carbamezepine, oral hypoglycemics, antidepressants, lipid-lowering drugs, known intolerance to conjugated estrogens, diabetes, untreated thyroid disease, clinical osteoporosis, and severe menopausal symptoms. In addition, the following exclusions applied to the challenge drugs: heavy alcohol or coffee use, significant cardiovascular disease, asthma, active peptic ulcer, hyperthyroidism, pyloric stenosis, narrow angle glaucoma, epilepsy, or current Axis I psychiatric disorders.

Upon meeting these criteria, subjects were approved for further screening at the University of Vermont (UVM) General Clinical Research Center (GCRC). After signing informed consent documents, subjects gave their medical history,and underwent physical and laboratory tests assessing hematopoietic, renal, hepatic and hormonal functions. Subjects were cognitively evaluated using the Mini Mental State Exam (MMSE; Folstein et al, 1975), Brief Cognitive Rating Scale (Reisberg et al, 1988), and the Mattis Dementia
Rating Scale (DRS, Jurica et al, 2001) to establish a Global Deterioration Scale score (GDS) that rates the degree of cognitive impairment (Reisberg et al, 1988). Subjects were required to have an MMSE score $\geqslant 27$, a DRS score of 123 or greater, and a GDS score of 1 or 2 .

Behavioral screening consisted of a partial Structured Clinical Interview for DSM-IV-TR (SCID; First et al, 2001) to establish the presence/absence of Axis I psychiatric disorders. In addition, subjects completed the Beck Depression Inventory (BDI). A cutoff score of 10 was used for the BDI, and subjects scoring over this criterion were discontinued from further participation. All subjects met these criteria for the cognitive and behavioral screening.

\section{Study Design}

The overall study design was a 2 (estrogen treatment: estradiol $v s$ placebo) by 5 (cholinergic challenge drug: $2.5 \mu \mathrm{g} / \mathrm{kg}$. SCOP, $5 \mu \mathrm{g} / \mathrm{kg}$ SCOP, $10 \mathrm{mg}$ MECA, $20 \mathrm{mg}$ MECA, and placebo) within-subjects crossover design.

\section{Estrogen Administration}

After meeting all inclusion criteria, subjects were randomly and blindly assigned to the estrogen or placebo condition for 3 months (Phase 1). In the estrogen condition, subjects took $1 \mathrm{mg}$ of oral $17 \beta$-estradiol (E2) per day for 3 months. In the placebo condition, subjects took similar appearing placebo pills for 3 months. After 3 months, subjects completed five challenge days (described below). They were then crossed over to the other condition, either placebo or estrogen, for another 3 months (Phase 2) and completed five more challenge days after those 3 months. After completion of the second phase of the study, all subjects took $10 \mathrm{mg}$ per day of medroxy-progesterone acetate for 12 days to produce sloughing of any endometrium that developed.

\section{Cholinergic Challenge Procedure}

After 3 months of estrogen or placebo treatment, subjects came in to the UVM GCRC for six sessions. At the first session, subjects were trained on the cognitive battery. They then took part in five cholinergic challenge study days. On each challenge day, subjects reported to the UVM GCRC by $0900 \mathrm{~h}$. Each participant performed a baseline motor skill sobriety test to have as a comparison to a second test before discharge in the afternoon. An intravenous (i.v.) line was inserted and blood was drawn for an estradiol assay. A double-blind, double placebo method of administration of the challenge drugs was followed. Subjects received one of the following medications: $2.5 \mu \mathrm{g} / \mathrm{kg}$ SCOP, $5 \mu \mathrm{g} / \mathrm{kg}$ SCOP, $10 \mathrm{mg}$ MECA, $20 \mathrm{mg}$ MECA, or placebo. SCOP was administered i.v. and MECA was administered orally. At time 0 , a pill containing one of the MECA doses or placebo was administered. After $30 \mathrm{~min}$, an injection of one of the SCOP doses or placebo was administered i.v. On each day only one of the drugs was active or both were placebo. The order of the drug administration across the 5 days was determined randomly. At 90 min after the injection and $2 \mathrm{~h}$ after oral pill administration, cognitive testing began at a running time of $120 \mathrm{~min}$. After the cognitive testing that 
took approximately $70 \mathrm{~min}$, subjects were given lunch. Vital signs and pupil diameter were assessed at seven time points throughout the session, at running times of $0,30,60,120$, 180,210 , and $240 \mathrm{~min}$. At the end of the study day, after passing the sobriety test to the satisfaction of the research nurse and covering physician, subjects were discharged.

\section{Cognitive Battery}

The cognitive testing battery was constructed to evaluate a number of cognitive domains potentially sensitive to cholinergic manipulation, as well as those affected by loss of and subsequent treatment with estrogen. These cognitive domains included tests of attention, verbal learning and memory, and nonverbal learning and memory. Before each of the challenge sessions, subjects were trained on the cognitive battery until they reached asymptotic performance to minimize learning or practice effects across sessions during each challenge phase.

\section{Attention}

The Critical Flicker Fusion (CFF) task (Kupke and Lewis, 1989), the Choice Reaction Time (CRT) task (Hindmarch, 1984), the Digit Symbol Substitution Test (DSST; Wechsler, 1981), the Divided Attention test (DA; Della Sala et al, 1995), and the Connors Continuous Performance Test (CPT; Conners, 1995) were used as the measures of attention. During the CFF task there were two different types of trials. In an ascending trial, the participant pressed a button that indicated when the frequency of flickering lights had increased to the point that the lights appeared to be no longer flickering but rather appeared continuously on ('fused'). The lights began flashing at a rate of $12 \mathrm{~Hz}$, increasing to $50 \mathrm{~Hz}$. In a descending trial, subjects indicated when the frequency of the apparently fused lights decreased such that lights appeared to be flickering. The participant was presented with three of each trial type. The dependent measure from the CFF was the median detection frequency across all trials.

The CRT task was a reaction time task in which subjects kept their index finger on a 'home' light-sensitive diode (LSD) until one of six LCD lights arrayed in a semicircle, approximately $25 \mathrm{~cm}$ from the 'home' key, was lit on the response box. The subject lifted her index finger and moved it to cover the LSD corresponding to the illuminated LCD. Performance measures included the median total reaction time (RT), the median recognition RT (time from stimulus onset to initiation of movement), and the median motor RT (time from initiation of movement to stimulus termination).

In the DSST, subjects were presented with nine numbers that corresponded to nine symbols. On the answer form, the subject was instructed to write the symbol that corresponded to each number and to complete as many as possible in $90 \mathrm{~s}$. The dependent measure on the DSST was the total correct completions.

In the DA task, the subject placed crosses on a series of interconnected boxes on a piece of paper. Simultaneously, a memory span test was performed, in which lists of numbers (length adjusted to individual maximums) were read to the subject and immediately recalled. Subjects performed each task separately, then together ( $2 \mathrm{~min}$ for each component).
Dependent measures for the DA task were the number of lists correctly recalled and the number of boxes completed on the maze during the single-task and dual-task portions of the test.

In the computerized CPT task, individual letters appeared on the computer screen for $300 \mathrm{~ms}$, with a response period of $2 \mathrm{~s}$ for 120 trials for a total time of $4.6 \mathrm{~min}$. Subjects were instructed to press a button when they saw an A followed by an X. The dependent measures for the CPT were hits, errors of omission and commission, and hit reaction time.

\section{Verbal Learning and Memory}

The Buschke Selective Reminding Task (SRT; Buschke, 1973), the Verbal Paired Associates Test (VPA; Wechsler, 1997), and the NYU Immediate Paragraph Recall (PR) test were used as measures of verbal learning and memory. In the SRT, subjects were read a list of 14 words, followed by an immediate recall trial. On subsequent trials (up to eight), subjects were immediately reminded of words they had failed to recall on the prior trial. Three measures were obtained from the SRT task: the total number of words recalled across all lists, recall consistency, and recall failure.

In the VPA, subjects were read a list of eight pairs of words. Then they were read the first word in each pair and asked to recall the associate. The list was read and recalled a maximum of six times. If the participant recalled all words on the list within the first three trials, the test was discontinued after three trials. Dependent measures for the VPA were the numbers correct for the strong and weak associates after three and six trials.

The final test of verbal memory was an NYU Immediate PR test. Subjects were read a short paragraph and then asked to retell the story. The dependent measure for the PR task was the number of information units recalled verbatim from memory.

\section{Nonverbal Learning and Memory}

The tests of nonverbal learning and memory included the Repeated Acquisition Task (RAT; Higgins et al, 1989) and the Benton Visual Retention Test (BVRT). There were two phases to the RAT, Performance and Learning. During the Performance phase, subjects pressed buttons 1,2 , or 3 to learn a 10-digit sequence of these numbers. Feedback was given with each button press. If a correct button press was made, a counter was incremented to move to the next number in the sequence. If an incorrect response was made, the computer screen timed out for $2 \mathrm{~s}$ and then returned to the current trial to allow the subject to make another selection. Subjects had learned the Performance sequence previously at the training session, and thus they were instructed to produce the same sequence at each testing session. The Learning phase was similar to the Performance phase, except that subjects were instructed to learn a new 10-digit sequence on each challenge day. Dependent measures from both phases were total number of errors, quarter life, and response rate (Newhouse et al, 1994).

In the BVRT, subjects were shown 10 geometric figures for $10 \mathrm{~s}$ each. Their task was to reproduce the figure immediately after viewing it. Dependent measures from the BVRT were the number of correct designs and errors. 
The cognitive tasks were administered in the following order: CFF, CRT, SRT, DA, RAT, PR, BVRT, DSST, VPA, and CPT. This administration order was the same for all subjects on all study days. A minimum of 10 equivalent versions of the testing forms (except for PR, where six forms were used) were created so that a new version of each test was available for each of the testing days (five challenge days for Phase 1 and Phase 2). These forms were counterbalanced across the study days for all subjects.

\section{Behavioral Measures}

After the cognitive battery was completed, subjects and the experimenters completed behavioral assessment measures. Subjects completed the Profile of Mood States (POMS; McNair et al, 1971), Stanford Sleepiness Scale (Hoddes et al, 1973), Subjective Visual Analogue Scale (SVAS; Newhouse et al, 1994), and a Physical Symptom Checklist (PSCL). The experimenter completed the Brief Psychiatric Rating Scale (BPRS; Overall and Gorham, 1993) and Objective Visual Analogue Scale (OVAS; Newhouse et al, 1994).

\section{Data Analysis}

Our main analysis of interest was whether 3 months of estrogen treatment would attenuate the negative effects of the antimuscarinic and antinicotinic cholinergic drugs on the cognitive tasks in this study. Four different approaches were taken to examine the data. First we calculated difference scores for the each of the dependent measures from each task. Performance on the cholinergic challenge placebo day was subtracted from performance on each of the other cholinergic challenge days (within a phase) in an effort to examine the specific effects of the cholinergic drugs on cognitive performance (Table 1). This was done to control for anticipated baseline differences in performance for different subjects in different phases of the study. Second, to demonstrate that the anticholinergic drugs produced impairments in the cognitive tasks, we examined one-sample $t$-tests for each dependent measure for each treatment by challenge drug dose condition. If the change in performance was greater than zero for any dependent measure, performance after anticholinergic challenge was different from the placebo challenge.

Third, we examined the ability of estrogen to attenuate the anticholinergic induced impairment on our tasks. The effects of SCOP and MECA were analyzed in separate models in order to examine the effects of estrogen to attenuate antimuscarinic and antinicotinic induced cognitive impairment separately. For the SCOP challenge days, 2 (estrogen treatment: E2 vs PLC) $\times 2$ (SCOP dose: $2.5 \mu \mathrm{g} / \mathrm{kg}$ vs $5.0 \mu \mathrm{g} / \mathrm{kg}$ ) mixed-model repeated-measures ANOVAs were run for each dependent measure. For the MECA challenge days, 2 (estrogen treatment: E2 vs PLC) $\times 2$ (MECA dose: $10 \mathrm{mg}$ vs $20 \mathrm{mg}$ ) mixed-model repeatedmeasures ANOVAs were run for each dependent measure. If estrogen attenuated the negative effects of the anticholinergic drugs on cognition, the main effects of estrogen would be seen in these analyses. Finally, we also examined the effects of age and years since menopause on each of the dependent measures with two separate ANCOVAs. The statistics are described for the dependent measures where the ANCOVAs produced different results than the ANOVAs in particular with regard to the specific effects of estrogen treatment.

Data from the behavioral measures and vital signs were analyzed separately for SCOP and MECA study days. For the SCOP study days, 2 (estrogen treatment: E2 vs PLC) $\times 3$ (challenge drug: $2.5 \mu \mathrm{g} / \mathrm{kg}$ SCOP, $5.0 \mu \mathrm{g} / \mathrm{kg}$ SCOP, PLC) mixed-model repeated-measures ANOVAs were run for each dependent measure. For the MECA study days, 2 (estrogen treatment: E2 vs PLC) $\times 3$ (challenge drug: $10 \mathrm{mg}$ MECA, $20 \mathrm{mg}$ MECA, PLC) mixed-model repeated-measures ANOVAs were run for each dependent measure. When significant effects of estrogen treatment were found in the behavioral analyses, we correlated those behavioral measures with the cognitive measures in order to further examine the relationship between the treatment and drug effects and cognitive and behavioral performances.

\section{RESULTS}

\section{The Effects of 3 Months of Estrogen Treatment on Baseline Cognitive Functioning}

We examined performance on the cognitive battery during the cholinergic challenge placebo days and compared the performance after 3 months of estrogen versus 3 months of placebo. Paired $t$-tests compared the performance on each task on the placebo challenge day after 3 months of estrogen vs 3 months of placebo treatment. All analyses showed no difference in performance between the estrogen and placebo days. Thus, there was no effect of 3 months of estrogen on performance of any of the tests in this battery. This finding is not unexpected given that we pretrained subjects on the battery so that their performance would be stable and not susceptible to learning or practice effects across the various study days. We further examined the data for practice or learning effects across treatment phase (1 or 2) and challenge days (1-5). There were no phase by day interactions and the patterns of means did not show increasing improvements across the study days and phase. Therefore, practice effects were not found on any of the cognitive tests.

\section{The Effects of Anticholinergic Drugs on Cognitive Performance}

In order to examine the ability of estrogen to attenuate the anticholinergic induced impairment on cognition, it is important to first demonstrate that SCOP and MECA reliably impaired performance on the tasks used in this study. Results of the one sample $t$-tests showed that SCOP significantly $(p<0.05)$ impaired performance on the CFF, CRT, DSST, CPT, DA, SRT, VPA, PR, and RAT Learning. MECA significantly $(p<0.05)$ impaired performance on the CRT, DSST, CPT, RAT performance, and BVRT. Details are available in Supplementary online information. The ability of estrogen to attenuate these performance impairments is described below.

Attention. The analysis of CFF performance during SCOP challenge revealed a main effect of estrogen treatment for the total median frequency across all trials, $F(1,12)=6.70$, 
Table I Performance Differences for Cognitive Tasks for Each Drug Challenge by Treatment Type

Cognitive construct Task Dependent variable

Attention

CRT Total (ms)

Recognition RT (ms)

Motor RT (ms)

DSST Number correct

CPT Hits (proportion correct)

Errors of omission (number of errors)

Hit RT (ms)

DA

Digit span — single task (number correct)

Digit span—dual task (number correct)

Maze — single task (number correct)

Maze — dual task (number correct)

Verbal memory

\section{SRT Total recall (number correct) \\ Recall consistency (number correct) \\ Recall failure (number of failures) \\ VPA \\ Three trials—hard (number correct) \\ Six trials - easy (number correct) \\ Six trials - hard (number correct)}

PR NYU scoring (number correct)

$\begin{array}{lll}\text { Non-verbal memory } & \text { RAT } & \text { Performance } \\ & \text { errors (percent errors) } \\ & \text { Quarter life }\end{array}$

Response rate (ms)
Treatment $2.5 \mu \mathrm{g} / \mathrm{kg}$ SCOP $5.0 \mu \mathrm{g} / \mathrm{kg}$ SCOP $10 \mathrm{mg}$ MECA $20 \mathrm{mg}$ MECA

\begin{tabular}{|c|c|c|c|c|}
\hline Placebo & $-2.38(0.70)$ & $-1.31(0.70)$ & $-0.62(0.52)$ & $-0.1(0.61)$ \\
\hline Estrogen & $-0.53(0.68)$ & $-0.28(0.68)$ & $-2.65(2.65)$ & $0.26(0.43)$ \\
\hline Placebo & $50.3(29.02)$ & 94.11 (21.92) & $49.04(17.40)$ & $36.07(17.40)$ \\
\hline Estrogen & $24.14(19.91)$ & $121.64(54.26)$ & $20.63(17.40)$ & $2.9(18.06)$ \\
\hline Placebo & $40.46(24.89)$ & 30.57 (20.97) & $9.59(7.81)$ & $-11.88(9.14)$ \\
\hline Estrogen & $25.49(|2.8|)$ & $76.13(28.41)$ & $-33.43(28.75)$ & 4.99 (12.49) \\
\hline Placebo & $21.33(18.64)$ & 37.02 (8.58) & $33.44(11.27)$ & $35.29(11.27)$ \\
\hline Estrogen & $8.63(9.75)$ & $27.39(21.52)$ & $9.89(11.27)$ & $-4.35(11.70)$ \\
\hline Placebo & $-4.07(2.09)$ & $-6.53(2.15)$ & $-2.07(1.25)$ & $-0.73(1.25)$ \\
\hline Estrogen & $-2.20(2.09)$ & $-6.47(2.09)$ & $-2.27(1.25)$ & $-2.60(1.25)$ \\
\hline Placebo & $-3.24(1.9)$ & $-3.25(1.37)$ & $-0.13(0.30)$ & $-0.13(0.30)$ \\
\hline Estrogen & $-0.97(0.37)$ & $-3.60(1.14)$ & $-0.3 \mid(0.3 \mid)$ & $-0.42(0.31)$ \\
\hline Placebo & $3.03(1.78)$ & $2.93(1.3)$ & $0.13(0.29)$ & $-0.07(0.29)$ \\
\hline Estrogen & $0.84(0.34)$ & $3.53(1.09)$ & $0.3(0.30)$ & $0.41(0.30)$ \\
\hline Placebo & $0.81(0.52)$ & $1.09(0.77)$ & $0.13(0.19)$ & $-0.33(0.27)$ \\
\hline Estrogen & $0.63(0.43)$ & $1.4(0.58)$ & $0.35(0.16)$ & $0.07(0.4 I)$ \\
\hline Placebo & 30.45 (9.96) & $22.09(21.46)$ & $56.92(12.57)$ & $52.53(|6.6|)$ \\
\hline Estrogen & $-14.15(10.90)$ & $-7.63(14.97)$ & 8.01 (9.63) & 23.27 (18.07) \\
\hline Placebo & $-2.5 \mid(4.77)$ & $0.47(4.77)$ & $1.94(3.37)$ & $2.74(3.22)$ \\
\hline Estrogen & $1.56(4.77)$ & $1.75(4.77)$ & $-0.15(3.94)$ & $2.32(5.98)$ \\
\hline Placebo & I.II (4.79) & $-3.62(4.7 \mathrm{I})$ & $-2.37(3.42)$ & $-1.69(3.42)$ \\
\hline Estrogen & $0.87(4.10)$ & $-0.30(5.62)$ & $5.12(3.42)$ & $0.16(3.42)$ \\
\hline Placebo & $-9.60(4.80)$ & $-2.87(3.33)$ & $-5.80(4.14)$ & $-1.93(3.55)$ \\
\hline Estrogen & $-21.20(3.59)$ & $-10.40(3.68)$ & $-8.33(3.92)$ & $-2.13(4.06)$ \\
\hline Placebo & $-6.47(4.45)$ & $-0.53(3.4 I)$ & $-3.00(3.09)$ & $0.67(3.16)$ \\
\hline Estrogen & $-14.13(3.30)$ & $-9.67(4.40)$ & $-3.80(2.58)$ & $-4.60(3.64)$ \\
\hline
\end{tabular}

$-7.87(3.88)$

$-20.53(5.10)$

$-2(2.39)$

$0.07(2.39)$

Estrogen

$-5.27(2.94)$

$-19.2(3.52)$

$-11.31(3.46)$

$-21.93(4.10)$

$-9.8(3.42)$

Estrogen

Placebo

$6.7 \mid(2.64)$

$-24.6(4.71)$

$11.6(2.55)$

Estrogen

$2.07(2.55)$

Placebo

$-0.60(0.34)$

Estrogen

Placebo

$-0.13(0.19)$

Estrogen

Placebo

Estrogen

Placebo

Estrogen

Placebo

Estrogen

$-1.00(0.64)$

$-0.93(0.64)$

$-0.6(0.26)$

$-0.27(0.43)$

$-1.60(0.83)$

$-0.73(0.93)$

$10.87(2.55)$

$-0.54(0.33)$

$-0.33(0.32)$

$-2.36(0.67)$

$-2.07(0.64)$

$-0.35(0.35)$

$-0.67(0.43)$

$-3.00(0.86)$

$-1.87(0.93)$

$-1.72(1.28)$

$-5.16(1.53)$

$-0.20(1.25)$

$-4.93(1.30)$

$-0.8(2.39)$

$-2.4(2.63)$

$-4.47(2.63)$

$1.8(1.61)$

$-1.8(1.09)$

$0.07(0.23)$

$-0.53(0.29)$

$0.13(0.57)$

$-0.73(0.57)$

$0.27(0.33)$

$-0.53(0.46)$

$0.20(0.76)$

$-1.00(0.76)$

$-0.45(1.12)$

$0.07(1.13)$

-1.27 (2.39)

$-1.13(2.63)$

$-1.6(2.63)$

$1.20(0.93)$

$2.00(1.38)$

$-0.47(0.27)$

$-0.27(0.26)$

$-0.07(0.57)$

$-0.27(0.57)$

$-0.53(0.3 \mathrm{I})$

$-0.27(0.27)$

$-0.47(0.76)$

$0.27(0.76)$

$-0.13(0.96)$

$0.40(1.65)$

Placebo

$3.13(2.55)$

$2.19(2.05)$

$-0.13(1.60)$

$-0.67(0.5 \mathrm{I})$

Estrogen

$-0.60(1.31)$

$4.7(2.63)$

$0.40(1.56)$

$-0.97(1.63)$

Placebo

$-0.86(7.46)$

$7.80(6.42)$

$-0.07(0.73)$

$-0.32(0.24)$

Estrogen

$3.24(4.66)$

$9.29(5.53)$

$0.17(0.70)$

$-0.67(0.73)$

Placebo

$0.06(0.09)$

$0.01(0.08)$

$7.63(9.61)$

$1.00(8.21)$

Estrogen

$-0.10(0.06)$

$0.02(0.12)$

$3.67(3.83)$

I.4I (4.61) 
Table I Continued

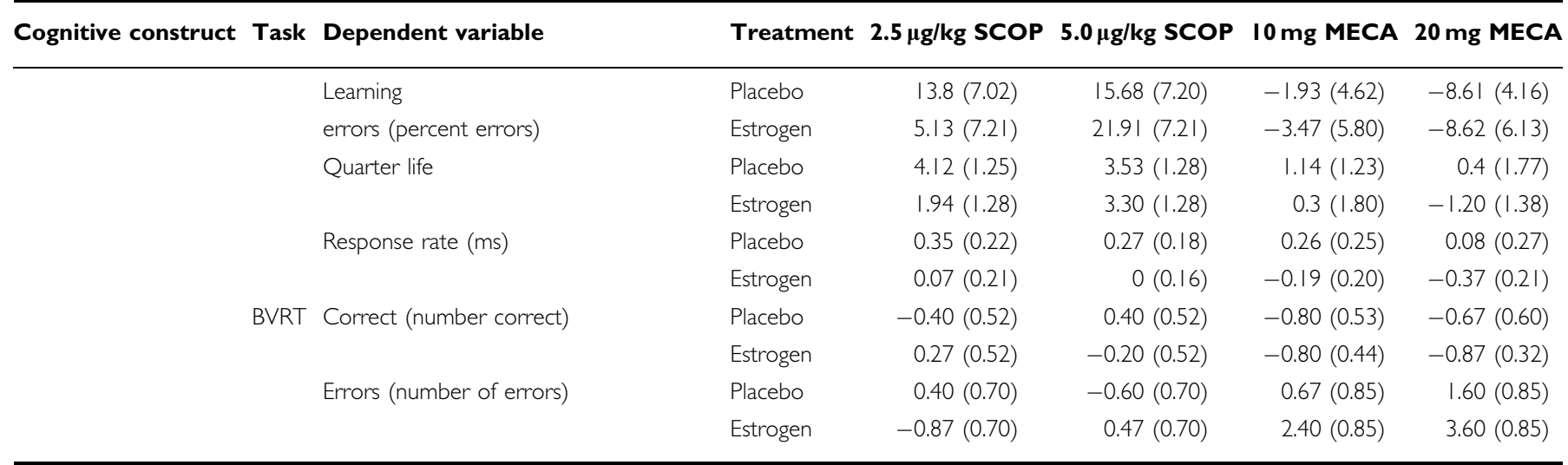

Numbers represent difference scores (drug minus placebo). Significant effects of estrogen treatment are indicated. See text for challenge drug effects. Cognitive tasks are Critical Flicker Fusion (CFF), Choice Reaction Time (CRT) test, Digit Symbol Substitution Test (DSST), Continuous Performance Test (CPT), Divided Attention (DA) test, Buschke Selective Reminding Test (SRT), Verbal Paired Associates (VPA), NYU Immediate Paragraph Recall (PR), Repeated Acquisition Test (RAT), and Benton Visual Retention Test (BVRT).

a Main effect of estrogen in the SCOP analysis for median total frequency on the CFF, $p<0.05$.

bMain effect of estrogen in the MECA analysis for motor RT on the CRT, $p<0.05$.

cMain effect of estrogen treatment in the SCOP and MECA analysis for the hit RT measure from the CRT, $p<0.05$ and $p<0.01$, respectively.

$p<0.05$, that showed that performance was less impaired on the CFF after 3 months of estrogen relative to placebo treatment during SCOP challenge (Figure 1). The ANCOVAs with age group and years since menopause as the covariates showed an effect of estrogen in each model, $F(1,12)=6.93$, $p<0.05$ and $F(1,12)=6.81, p<0.05$, respectively. CFF performance was better on the estrogen treatment days relative to the placebo treatment days for total median frequency. For the analysis of CFF performance during the MECA challenge, no significant effects were found.

For the CRT task, performance on SCOP challenge days showed no drug effects on the total median RT but for the median recognition RT there was an effect of SCOP dose, $F(1,14)=5.51, p<0.05$, that showed that recognition RT was longer in the high-dose SCOP conditions. For the median motor RT, the SCOP dose effect approached significance, $F(1,14)=4.12, p=0.06$. The pattern of means indicated that motor RTs were longer in the high-dose SCOP condition. There was no effect of estrogen treatment on RT in the SCOP analysis.

During the MECA challenge there was an effect of estrogen treatment on median motor RT, $F(1,12)=7.71, p<0.05$, which showed that motor RTs were shorter on the MECA challenge days after three months of estrogen treatment relative to placebo treatment (Figure 2). The ANCOVA with years since menopause as the covariate revealed similar results for estrogen treatment, $F(1,12)=7.15, p<0.05$. Additionally, the one-sample $t$-tests showed that, for the motor RT component, performance after estrogen treatment during MECA challenge was no different from performance during placebo challenge. Thus, estrogen improved performance on the motor RT of the CRT during MECA challenge. There was little effect of either dose of MECA on total RT or recognition RT.

For the DSST, there was a trend toward a main effect of SCOP dose, $F(1,14)=3.62, p=0.08$. The data pattern indicated that there were fewer correct completions for the high SCOP condition relative to the low SCOP condition. There were no effects of MECA on the DSST, and there were no effects of estrogen treatment under either drug challenge.

The analyses of the CPT data showed similar patterns for the SCOP and MECA challenge days. For both analyses, there was an effect of estrogen condition on hit RT, SCOP: $F(1,14)=7.69, p<0.05$, and MECA: $F(1,14)=9.71, p<0.01$ (Figure 3). There were very few errors of omission or commission overall; thus, it is reasonable to examine hit RT as a measure of the overall performance on this task. Estrogen treatment resulted in shorter hit RTs relative to the placebo treatment conditions; thus, estrogen attenuated the impairing effects of the anticholinergic drugs on hit RT. Estrogen treatment resulted in an improvement in throughput on the CPT task as the speed of hit performance was improved without affecting accuracy, and thus there was no speed-accuracy tradeoff.

The ANOVAs for the DA task showed similar patterns for the SCOP and MECA days. There were main effects of SCOP dose, $F(1,14)=12.89, p<0.01$, and MECA dose, $F(1,14)=$ $6.48, p<0.05$, for the number of boxes completed in the dual-task condition that showed that subjects completed fewer boxes on the high-dose days. Additionally, both of the ANCOVAs using age and years since menopause as the covariates found a trend toward a main effect of estrogen, $F(1,13)=3.76, p=0.07$ and $F(1,13)=3.78, p=0.07$, respectively. However, fewer boxes were completed during the single task maze completion test on estrogen compared to placebo. Thus, estrogen did not improve performance on the DA task during anticholinergic drug challenge.

To summarize the findings from the attention tasks, the effect of estrogen treatment was most clearly seen on CFF during SCOP challenge, on the motor RT from the CRT under MECA challenge and on the hit RT measure from the CPT under both SCOP and MECA challenges. In all cases estrogen treatment resulted in improved performance during anticholinergic drug challenge relative to placebo treatment. 


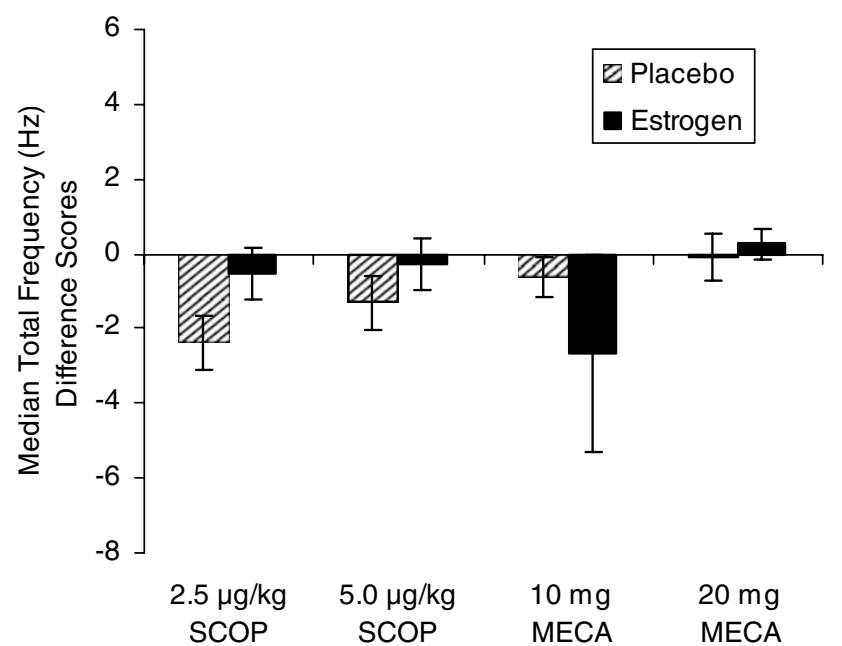

Figure I Difference scores for the total frequency measure on the CFF on SCOP and MECA challenge days minus placebo day performance. Results showed an effect of estrogen during SCOP study days only, $F(I, I 2)=6.70, p<0.05$. Estrogen attenuated the negative effects of SCOP on CFF performance.

Verbal learning and memory. The data from the SRT during SCOP challenge days showed an effect of SCOP dose on all three dependent measures, recall across eight trials, $F(1,14)=12.95, \quad p<0.01$, recall consistency across eight trials, $F(1,14)=18.18, p<0.001$, and recall failure across eight trials, $F(1,14)=7.09, p<0.05$. For all measures, the high-dose SCOP condition impaired performance relative to the low-dose SCOP condition. Estrogen treatment did result in a reduction in recall failure in the low SCOP condition after estrogen treatment relative to placebo treatment (Figure 4), although this did not reach statistical significance.

SRT performance on the MECA study days showed no effects of either drug condition on eight trial recall or eight trial consistency. On the eight trial recall failure measure, there was an interaction of estrogen treatment and MECA dose, $F(1,14)=4.82, p<0.05$. Post hoc comparisons showed no differences between the low and high MECA doses on the placebo days, $p>0.10$ (Figure 4).

There were no significant effects of any of the drug conditions on the VPA performance. There was a trend toward a main effect of SCOP dose for the hard pairs after three trials, $F(1,14)=3.66, p=0.08$. The pattern of means indicated that fewer word pairs were remembered in the high SCOP condition relative to the low SCOP condition.

For PR performance on the SCOP challenge days, there was an effect of SCOP dose, $F(1,14)=52.28, p<0.001$, that showed that subjects recalled fewer story units during the high-dose SCOP testing session relative to the low-dose SCOP session. There were no effects of either dose for the MECA study days. There were no effects of estrogen treatment seen in this task.

To summarize the results from the verbal memory tasks, an estrogen effect was seen in the recall failure measure of the SRT during MECA challenge reducing the effect of MECA. Although the effects of SCOP on verbal memory were more prevalent, effects of estrogen appeared to attenuate the effects of low-dose SCOP, but the effects did not reach statistical significance.

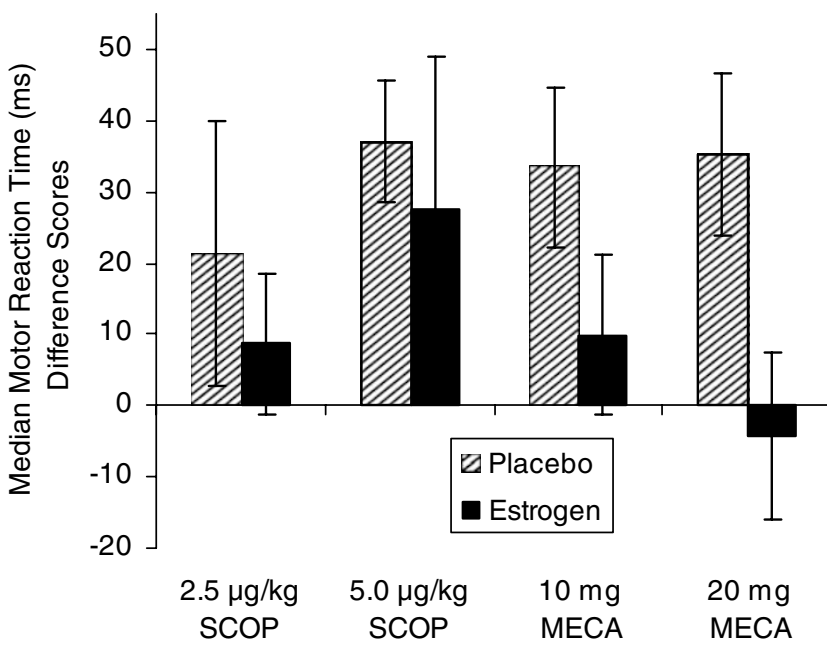

Figure 2 Difference scores for the median motor RT on the CRT on SCOP and MECA challenge days minus placebo day performance. After estrogen treatment, the RT cost of MECA challenge was significantly less than after placebo treatment, $F(I, I 2)=7.71, p<0.05$

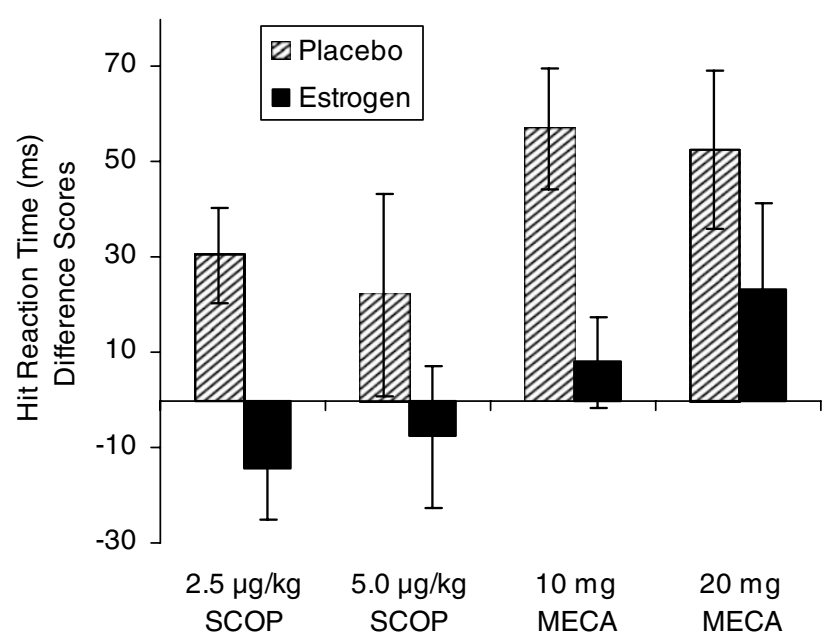

Figure 3 Difference scores for hit RT on the CPT during SCOP and MECA challenge days minus placebo day performance. Estrogen attenuated the negative effects of both SCOP, $F(I, I 4)=7.69, p<0.05$, and MECA, $F(I, \mid 4)=9.71, p<0.01$, on hit RT.

Non-verbal learning and memory. Data were analyzed separately for the Performance and Learning phases of the RAT. For the Performance phase, there were no significant effects during the SCOP challenge days. During the MECA challenge days, there was a main effect of MECA dose on the response rate measure in the Performance phase, $F(1,14)=6.16, p<0.05$. Subjects' responses were slower on the high-dose MECA day relative to the low-dose MECA day.

For the RAT Learning phase, there were no significant drug effects during the SCOP challenge days. For the MECA challenge days, there was a trend toward a main effect of MECA dose for the percent total errors, $F(1,14)=3.91$, $p=0.07$, that showed that subjects made more errors on the high-dose MECA relative to the low-dose MECA. The effect of MECA dose for the response rate measure also approached significance, $F(1,14)=4.15, p=0.06$. Subjects 
responded slower on the high-dose MECA days relative to the low-dose MECA days. Additionally, the ANCOVA using years since menopause as the covariate showed a strong trend toward an effect of estrogen treatment, $F(1,13)=4.08$, $p=0.06$. Estrogen speeded response rate relative to the placebo treatment condition during MECA challenge.

Data from the BVRT during SCOP challenge showed an effect of SCOP dose on the error measure, $F(1,14)=6.83$, $p<0.05$, where subjects made more errors on the high SCOP study days. There were no effects for the MECA study days from the ANOVAs. There were also no effects of estrogen treatment on BVRT performance.

\section{Behavioral Measures}

Questionnaires assessing mood and physical symptoms were administered after cognitive testing was completed to examine whether there were any negative mood or physical symptoms as a result of the estrogen treatment and challenge drugs. There were statistically significant, but clinically minor, effects of SCOP and MECA that can be summarized as expected effects from the anticholinergic drugs. Estrogen treatment did little to influence subjective or objective behavioral ratings.

The SVAS scales assessed Anxiety, Mood, Alertness, Comfort, Fear, Irritability, Hunger, and Interest. An interaction of estrogen and SCOP dose as well as a main effect of estrogen treatment were found for the Interest measure on the SVAS during SCOP study days, $F(1,14)=12.81, p<0.01$, and $F(2,22)=4.19, p<0.05$, respectively. Subjects reported slightly less interest on study days after estrogen treatment. Interest declined more with increasing SCOP dose during estrogen treatment compared to placebo treatment. No effects of estrogen treatment were seen on the SVAS during the MECA study days.

There was a main effect of estrogen treatment on the Blurry Vision measure from the PSCL on the SCOP study days, $F(1,14)=9.45, p<0.01$. Subjects rated somewhat more

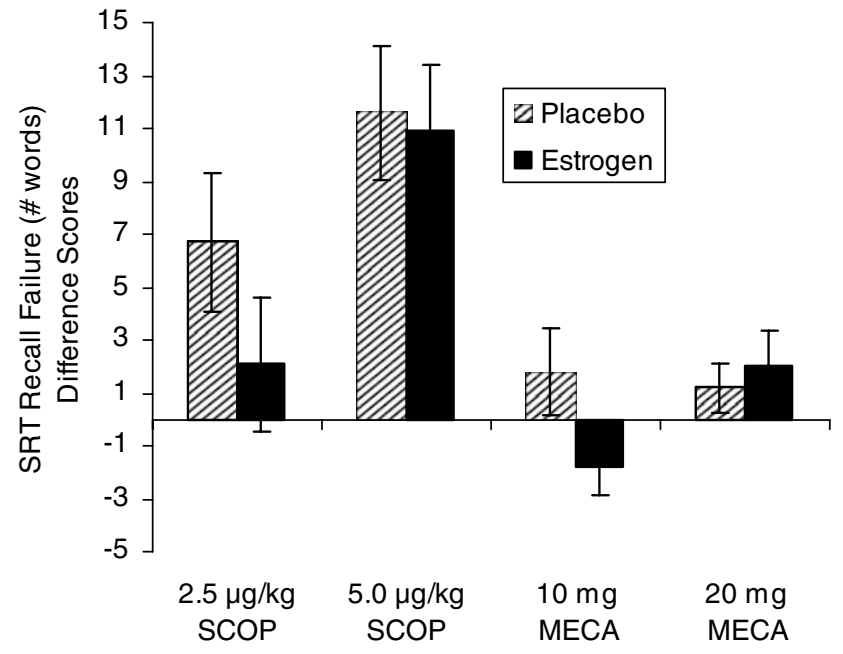

Figure 4 Difference scores for recall failure on the SRT during SCOP and MECA challenge days minus placebo day performance. Estrogen attenuated the negative effects of MECA during the low-dose MECA, $F(I, \mid 4)=4.82, p<0.05$. blurred vision after estrogen treatment compared to placebo treatment; however, the magnitude of this effect was very small. There were no effects of estrogen treatment found on the PSCL on MECA study days.

The OVAI assessed Drowsiness, Restlessness, Disorientation, Impaired Speech, Euphoria, Irritability, Sweating, GI Distress, Motor Incoordination, Fatigue, Depression, Anxiety, and Alertness. On the OVAI, there was a main effect of estrogen on the Fatigue measure, $F(1,9)=6.17, p<0.05$, that showed that subjects were rated by the experimenter as slightly more fatigued on study days in the estrogen treatment phase relative to the placebo treatment phase. No estrogen effects were found for the MECA study days on the OVAI.

No effects of estrogen treatment were found for the POMS, Stanford Sleepiness Scale, or the BPRS.

Correlations were examined between the behavioral ratings that showed effects of estrogen treatment (Interest from the SVAS, Blurry Vision from the PSCL, and Fatigue from the OVAI) and the cognitive measures. Significant correlations were seen for these three behavioral measures with some cognitive measures; however, no consistent relationships were seen. In most cases the expected relationships were found during SCOP challenge days with increases in fatigue related to decreases in performance. Additionally, the behavioral changes across estrogen treatment conditions were small and did not significantly affect the performance.

To summarize the results from the behavioral ratings, estrogen treatment affected very few of the subjective or objective ratings and changes to these ratings only inconsistently correlated with cognitive performance. Any beneficial effects of estrogen treatment on cognitive performance were therefore not a result of improved arousal or mood due to estrogen treatment.

\section{Vital Signs}

Blood pressure, pulse, and pupil diameter were monitored at seven time points throughout the challenge days. Analyses were conducted on the maximum change score from the baseline measurement for each variable. On the SCOP challenge days, there were no significant effects of challenge drugs or estrogen treatment for the maximum change from baseline for any of the vital signs measures. On MECA study days, there was a main effect of challenge drug on systolic blood pressure, $F(2,28)=5.72, p<0.01$, that showed the expected lower systolic blood pressure on MECA study days relative to placebo. There was also a main effect of challenge drug on pupil diameter, $F(2,28)=5.72$, $p<0.01$, that showed that pupil diameter increased with increasing MECA dose. Estrogen treatment did not affect any of the vital signs measures during MECA challenge days.

\section{DISCUSSION}

The results from this study strongly suggest that even a relatively short-duration ( 3 months) and low-dose $(1 \mathrm{mg})$ estradiol administration to normal postmenopausal women was sufficient to blunt the effects of SCOP, a muscarinic 
antagonist, and MECA, a nicotinic antagonist, on measures of psychomotor function and attention, as well as other measures with a speed component. Additionally, these effects of estrogen were not related to a reduction of sedation, and were thus unlikely to be the result of a direct antagonism of estrogen on the pharmacologic effects of SCOP and MECA. Thus, the results of this study suggest that estrogen may interact with the cholinergic system to provide a buffer against declining efficiency in cognitive processing as a result of the loss of cholinergic tone with aging. While studies have shown the importance of estrogen-cholinergic system interaction on cognition in animals, this is the first study to demonstrate this relationship in humans. The exact cellular level interactions can only be speculated based on the data from this study, but prior research provides a basis for hypothesis generation regarding the cellular level interaction between estrogen and the cholinergic system. Proposals regarding estrogen's effects on cognition and its interactions with the cholinergic system are considered below.

\section{Estrogen, the Cholinergic System, and Cognition}

The effects of estrogen were most prominently seen on anticholinergic-induced changes in measures of psychomotor function and attention. Estrogen was additionally beneficial in speeding response times on some of the attention measures. The interaction of estrogen with the cholinergic system had a general effect on improving RT and cognitive efficiency. The finding that estrogen interacted with the cholinergic system to influence attentional performance supports findings from the primate literature (Tinkler and Voytko, 2005) showing that estrogen affects cholinergic functioning and attentional processing. The finding that estrogen did not attenuate the effects of SCOP and MECA on the accuracy measures of learning and memory was not predicted based on the prior research on the effects of ET on cognition (Sherwin, 1988; Verghese et $a l, 2000)$. However, the patterns of means across many dependent measures indicated that estrogen may be potentially protective against anticholinergic cognitive impairment on our tasks, but a small sample size reduced the statistical power to detect these effects (although the learning and memory tasks used in this study were generally sensitive to cholinergic manipulation).

The effects of estrogen were only seen during anticholinergic challenge and not on the placebo days. Similar to other studies, we found no beneficial effect for estrogen alone (Binder et al, 2001; Ditkoff et al, 1991; Janowsky et al, 2001). In this study, while we did train subjects prior to challenge days, there are implications for other studies looking at the effects of estrogen on cognition. Studies that do not find an effect of estrogen may be explained by performance issues with asymptotic or ceiling level performance by cognitively normal subjects. If estrogen has an effect on the cholinergic system, then subjects similar to the women in our study, who presumably have 'normal' cholinergic systems, will only show a visible effect if that system is stressed by administering anticholinergic drugs.

Several decades of research support the critical role of CNS cholinergic systems in cognition, particularly in learning and memory formation and attention (ie Caine et al, 1981; Drachman and Leavitt, 1974; Ghoneim and Mewaldt, 1977; Newhouse et al, 2001; Peterson, 1977; Sitaram et al, 1978). Sarter et al (2003) have suggested that cholinergic system functions are primarily attentional in nature. The cholinergic system supports the detection of external signals (bottom-up information processing) as well as processing of task-relevant and knowledge-based detection processes (top-down information processing; Sarter et al, 2005). Recruitment of the cholinergic system appears to increase with increasing cognitive demands and may reflect effortful processing (Sarter et al, 2005). The data from the current study are consistent with Sarter's hypothesis that cholinergic systems mediate attentional effects.

The current study also supports findings from an important series of studies by Voytko and colleagues examining the effects of ovariectomy and estrogen replacement on cholinergic-related cognitive performance in rhesus monkeys (Tinkler et al, 2004; Tinkler and Voytko, 2005; Voytko, 2002; Voytko and Tinkler, 2004). In these studies, SCOP effects on RT in visual spatial attention tasks were sensitive to the presence or absence of estradiol, suggesting that estradiol uses the cholinergic system to modulate visual-spatial attention performance. ET in surgically menopausal monkeys interacted with the cholinergic system to maintain visual-spatial attention and not visual-spatial memory. Interestingly, E2 administration in studies by Voytko and colleagues showed RT performance enhancements that were in part related to the attentional component of the task, not simply the motor performance requirements. This finding confirms the proposal that attentional load determines the degree of estrogen - cholinergic, interaction which is supported by the work of Sarter et al (2005) and Bentley et al $(2003,2004)$. Their results are similar to the findings of the current study that found effects of the interaction of estrogen and the cholinergic system on measures of attention and not memory. Our study is the first to demonstrate experimentally in humans the effects of E2 and its interaction with the cholinergic system and the effects of the interaction on specific cognitive processes.

The mechanism responsible for resistance to anticholinergic induced impairment cannot be definitively established from this experiment, but may include the neurotrophic effects of estrogen on basal forebrain cholinergic neurons mediated through NGF (Lee and McEwen, 2001; Toran-Allerand, 1996). For example, the early dysregulation of basal forebrain cholinergic neurons mediates the early attentional and performance impairment in AD (Sarter and Bruno, 2004), and loss of neurotrophic receptors such as tyrosine kinase (trkA) may in part underlie the neurodegeneration of these basal forebrain cholinergic neurons and subsequent loss of cholinergic capability, leading to cognitive impairment in Mild Cognitive Impairment (MCI) and later $\mathrm{AD}$ (Sarter et al, 2005). Intriguingly, there is evidence that estrogen specifically regulates the expression of neurotrophic receptors like trkA (Toran-Allerand, 1996). E2 appears to be able to directly regulate NGF receptor expression, including both trkA and the low-affinity receptor p75ntr, as well as the number of E2 receptors (Gibbs and Pfaff, 1992; Sohrabji et al, 1994a, b). Following neuronal injury or E2 deficiency 
(eg, menopause), there is an apparent reversion of E2 receptor regulation via neurotrophins back to a developmental pattern in which responsiveness to E2 can once again be demonstrated (Lee and McEwen, 2001; ToranAllerand, 1996). Enhanced growth, transcription, and proliferation of these neurons and their connections may follow, providing some resistance to the antagonistic effects of anticholinergic drugs.

This relationship between estrogen and neurotrophic factors provides a biologically plausible mechanism, whereby the loss of estrogen support may contribute to the dysfunction of basal forebrain cholinergic neurons associated with aging and neurodegenerative disorders and a mechanism to potentially explain the salutary effects of E2 administration on cholinergic-related cognitive performance as detected by the antagonist model in this study. These results also suggest that the basal forebrain cholinergic system may still be plastic enough to respond to hormonal stimulation even many years after those hormones are reduced or absent. Even short periods of estrogen administration may be sufficient to provide longterm protection by enhancement of cholinergic function. How long such protection may last and what effects progestins have will require further research.

Another interpretation of the data from the current experiment is that estrogen administration alters the pharmacokinetics or metabolism of SCOP. We believe this to be less likely due to the physiologic levels of the estrogen dosage used in this study, and that reports of subjective effects of the drugs did not differ for the most part between estrogen and placebo treatment phases. Alternatively, the presence of estrogen may alter signal transduction at the muscarinic receptor by influencing receptor binding and second messenger systems. The lack of a significant estrogen treatment effect on vital signs and pupil diameter tends to suggest that simple pharmacokinetic or pharmacodynamic effects are less likely to explain the estrogen treatment effects.

The findings from this study provide support for the general slowing account of cognitive aging (Salthouse, 1996) that provides a bridge between psychological theorizing about aging and cognition and the biological underpinnings. Under this model, the age-related variance in higher level cognitive tests like learning and memory can be explained by age variance in lower level processes like processing speed and reaction time. We have shown that estrogen can buffer the effects of a temporary cholinergic lesion on the arousal, attention, and speed tasks. Other studies have shown a positive correlation between cognitive efficiency or processing speed and memory performance (see Verhaeghen and Salthouse (1997) for a meta-analysis). Thus, before menopause, estrogen's effect on cognition may be to maintain overall cognitive efficiency, and when given to postmenopausal women it may buffer the negative effects of aging that lead to reduced cognitive processing efficiency. Additionally, our data suggest that the general aging effects on cognitive slowing are probably produced by age-related changes in the cholinergic system.

Three possible explanations arise for the lack of an effect of estrogen on the learning and memory measures. The first is that the tasks examined in this study do not afford the necessary sensitivity to measure the effects of the interaction of estrogen and the cholinergic system. The response time measures in the attention tasks most reliably showed the effects of estradiol. With the exception of the RAT, none of the learning and memory tasks included a response time measure. If the learning and memory tasks are sufficiently complex, requiring a number of overlapping cognitive operations for correct performance, the effect of estrogen administration may have been missed as a result of task complexity. For example, good performance on the SRT requires subjects to simultaneously encode, store, and retrieve information. Cholinergic systems have been shown to be important for encoding, but not retrieval (Newhouse et al, 1994), and a task with many simultaneous cognitive operations may not have been useful for detecting the more subtle effects of estrogen on cholinergic system modulation. A second possible explanation for the lack of significant findings on the learning and memory measures includes differences in the length of treatment in this study relative to the prior literature. Estrogen treatment for 3 months in women who have undergone normal menopause may not be enough time to affect the accuracy measures on these tasks. Finally, differences in the estrogen dose and the formulation of estrogen across studies may be the explanation for different findings on the learning and memory measures across studies. The effects of estrogen administration on nicotinic blockade were more limited because MECA produced significant impairment predominantly on the motor reaction time. This may indicate differences in the types of cognitive tasks and/or operations that are sensitive to muscarinic versus nicotinic blockade.

Future studies may include different cognitive measures, increase the estrogen dose or use of an estrogen with a different route of administration (patch $v s$ pills). Preliminary data from our laboratory suggest that administration of $2 \mathrm{mg}$ per day of E2 for 3 months to older women may potentially interact more strongly with the learning and memory measures (Hancur et al, 2003).

\section{Clinical Implications}

If confirmed, these data suggest that the cholinergic system in humans may be an important system for cognition that is sensitive to hormonal state. The cholinergic system is inarguably the most prominent neurochemical system involved in age- and disease-related deterioration in cognitive performance, especially in neurodegenerative dementias, for example, AD. Declining function or dysfunction of the basal forebrain cholinergic system may weaken both signal-driven and top-down or task-related attentional processing (Sarter et al, 2005), increasing errors and distractibility. Such changes may be responsible in part for the perception of attentional impairment by perimenopausal and postmenopausal women, and more importantly may interact with more severe cholinergic dysfunction secondary to developing neurodegenerative processes such as MCI or early AD.

Further studies will help to define the limits of estradiol or related compounds' abilities to manipulate the cholinergic system in normal women as well as in women with neurodegenerative disorders. Additional follow-up work is needed to examine precisely the impact of attentional factors on estradiol-cholinergic interactions, evaluate 
estrogen plus progesterone and estrogen antagonist effects on cholinergic system-modulated performance, investigate the parametric effects of estradiol dose and duration, and examine the functional neuroanatomy underlying these effects.

\section{SUMMARY}

This study investigated the interaction of estrogen and cholinergic system functioning and the effects of this interaction on cognitive task performance in healthy older women. Normal postmenopausal women were placed on $1 \mathrm{mg}$ 17- $\beta$ estradiol or placebo for 3 months each, followed by cholinergic challenge sessions, where they were administered one of two doses of the antimuscarinic drug SCOP or the antinicotinic drug MECA or placebo. Results showed that estrogen pretreatment attenuated the anticholinergic drug-induced impairments on tests of attention and tasks with speed components. Effects on tasks of episodic memory were suggestive of a similar protective effect, though not definitive. Data showing reduced anticholinergic effects on attentional performance in women while administering estradiol are consistent with studies and a variety of species that suggest that cholinergic systems modulate attentional processing of stimuli, and that estrogen status can affect cholinergic system activity and perhaps integrity.

\section{ACKNOWLEDGEMENTS}

This work was supported by R01 AG021476, AA IIRG-991811, and FAHC POR grant to PN, F32 AG023430 to JD, and NCRR-00109. We thank the research nursing staff of the University of Vermont GCRC for their hard work and support of this study and our volunteers for their dedication to clinical research.

\section{REFERENCES}

Barrett-Connor E, Kritz-Silverstein D (1993). Estrogen replacement therapy and cognitive function in older women. J Am Med Assoc 269: 2637-2641.

Bartus RT, Dean III RL, Beer B, Lippa AS (1982). The cholinergic hypothesis of geriatric memory dysfunction. Science 217: 408-417.

Bentley P, Husain MK, Dolan RJ (2004). Effects of cholinergic enhancement on visual stimulation, spatial attention, and spatial working memory. Neuron 41: 969-982.

Bentley P, Vuilleumier P, Thiel CM, Driver J, Dolan RJ (2003). Effects of attention and emotion on repetition priming and their modulation by cholinergic enhancement. J Neurophysiol 90: 1171-1181.

Bi R, Foy MR, Vouimba RM, Thompson RF, Baudry M (2001). Cyclic changes in estradiol regulate synaptic plasticity through the MAP kinase pathway. Proc Nat Acad Sci 98: 13391-13395.

Binder EF, Schechtman KB, Birge SJ, Williams DB, Kohrt WM (2001). Effects of hormone replacement therapy on cognitive performance in elderly women. Maturitas 38: 137-146.

Buschke H (1973). Selective reminding for analysis of memory and learning. J Verbal Learn Verbal Behav 12: 543-550.

Caine ED, Weingartner H, Ludlow CL, Cudahy EA, Wehry S (1981). Qualitative analysis of scopolamine-induced amnesia. Psychopharmacology 74: 74-80.
Conners CK (1995). The Continuous Performance Test. MultiHealth Systems: Toronto.

Daniel J, Dohanich G (2001). Acetylcholine mediates the estrogeninduces increase in NMDA receptor binding in CA1 of the hippocampus and the associated improvement in working memory. J Neurosci 21: 6949-6956.

Della Sala S, Baddeley A, Papagno C, Spinnler H (1995). Dual-task paradigm: a means to examine the central executive. Ann NY Acad Sci 769: 161-171.

Ditkoff EC, Crary WG, Cristo M, Lobo RA (1991). Estrogen improves psychological function in asymptomatic postmenopausal women. Obstetr Gynecol 78: 991-995.

Drachman D (1977). Memory and cognitive function in man: does the cholinergic system have a specific role? Neurology 27: 783-790.

Drachman D, Leavitt J (1974). Human memory and the cholinergic system. A relationship to aging? Arch Neurol 30: 113-121.

Duka T, Tasker R, McGowan JF (2000). The effects of 3-week estrogen hormone replacement on cognition in elderly healthy females. Psychopharmacology 149: 129-139.

Farr SA, Banks WA, Morley JE (2000). Estradiol potentiates acetylcholine and glutamate-mediated post-trial memory processing in the hippocampus. Brain Res 864: 263-269.

First MB, Spitzer RI, Gibbon M, Williams JBW (2001). SCID-I/P, 2/2001 edn. American Psychiatric Press Inc.: Washington, DC.

Folstein MF, Folstein SE, McHugh PR (1975). 'Mini-mental state': a practical method for grading the cognitive state of patients for the clinician. J Psychiatr Res 12: 189-198.

Gardiner SA, Morrison MF, Mozley PD, Mozley LH, Brensinger C, Bilker W et al (2004). Pilot study on the effect of estrogen replacement therapy on brain dopamine transporter availability on healthy, postmenopausal women. Am J Geriatr Psychiatry 12: 621-630.

Ghoneim MM, Mewaldt SP (1977). Studies on human memory: the interactions of diazepam, scopolamine, and physostigmine. Psychopharmacology 52: 1-6.

Gibbs RB (1996). Expression of estrogen receptor-like immunoreactivity by different subgroups of basal forebrain cholinergic neurons in gonadectomized male and female rats. Brain Res 72: $61-68$.

Gibbs RB, Burke AM, Johnson DA (1998). Estrogen replacement attenuates effects of scopolamine and lorazepam on memory acquisition and retention. Hormones Behav 34: 112-125.

Gibbs RB, Pfaff DW (1992). Effects of estrogen on fimbria/fornix transection on p75NGFR and ChAT expression in the medial septum diagonal band of Broca. Exp Neurol 116: 23-39.

Gibbs RB, Wu D, Hersh LB, Pfaff DW (1994). Effects of estrogen replacement on the relative levels of choline acetyltransferase, trkA, and nerve growth factor messenger RNAs in the basal forebrain and hippocampal formation of adult rats. Exp Neurol 129: $70-80$

Grady D, Yaffe K, Kristof M, Lin F, Richards C, Barrett-Connor E (2002). Effect of postmenopausal hormone therapy on cognitive function: the heart and estrogen/progestin replacement study. Am J Med 113: 543-548.

Halbreich U, Lumley LA, Palter S, Manning C, Gengo F, Joe S-h (1995). Possible acceleration of age effects on cognition following menopause. J Psychiatr Res 29: 153-163.

Hancur CA, Newhouse PA, Naylor MR, Schoenfeld JD, Sites CK (2003). Effects of varying estrogen dose on anti-cholinergic induced cognitive impairment in post-menopausal women. Biol Psychiatry 53: 139S.

Higgins ST, Woodward BM, Henningfield JE (1989). Effects of atropine on the repeated acquisition and performance of response sequences in humans. J Exp Anal Behav 51: 5-15.

Hindmarch I (1984). Psychological performance models as indicators of the effects of hypnotic drugs on sleep. Psychopharmacology S1: 58-68. 
Hoddes ElZ V, Smythe H, Phillips R, Dement W (1973). Quantification of sleepiness: a new approach. Psychophysiology 10: $431-436$.

Jacobs DM, Tang MX, Stern Y, Sano M, Marder K, Bell KL et al (1998). Cognitive function in nondemented older women who took estrogen after menopause. Neurology 50: 368-373.

Janowsky JS, Leigland LA, Orwoll ES, Stevens AS (2001). fMRI of age and estrogen effects on cognition in older women. Annual Meeting of the Society for Neuroscience.

Jurica PJ, Leitten CL, Mattis S (2001). Dementia Rating Scale-2. Psychological Assessment Resources Inc.: Lutz, FL.

Kritzer M, Kohama S (1999). Ovarian hormones differentially influence immunoreactivity for dopamine beta-hydroxylase, choline acetyltransferase, and serotonin in the dorsolateral prefrontal cortex of adult rhesus monkey. J Comp Neurol 409: 438-451.

Kupke T, Lewis R (1989). Relative influence of subject variables and neurological parameters on neuropsychological performance of adult seizure patients. Arch Clin Neuropsychol 4: 351-363.

Lee SJ, McEwen BS (2001). Neurotrophic and neuroprotective actions of estrogens and their therapeutic implications. Annu Rev Pharmacol Toxicol 41: 569-591.

Luine VN, Khylchevskaya RI, McEwen BS (1975). Effect of gonadal steroids on activities of monoamine oxidase and choline acetylase in rat brain. Brain Res 86: 293-306.

Luine VN, Renner KJ, McEwen BS (1986). Sex-dependent differences in estrogen regulation of choline acetyltransferase are altered by neonatal treatments. Endocrinology 119: 874-878.

McGaughy J, Sarter M (1999). Effects of ovariectomy, 192 IgGsaporin-induced cortical cholinergic deafferentation, and administration of estradiol on sustained attention performance in rats. Behav Neurosci 113: 1216-1232.

McMillan PJ, Singer CA, Dorsa DM (1996). The effects of ovariectomy and estrogen replacement on trkA and choline acetyltransferase mRNA expression in the basal forebrain of the adult female Sprague-Dawley rat. J Neurosci 16: 1860-1865.

McNair DM, Lorr M, Droppleman LF (1971). Profile of Mood States. Educational and Industrial Testing Service: San Diego, CA.

Newhouse PA, Potter A, Corwin J, Lenox R (1992). Acute nicotinic blockade produces cognitive impairment in normal humans. Psychopharmacology 108: 480-484.

Newhouse PA, Potter A, Corwin J, Lenox R (1994). Age-related effects of the nicotinic antagonist mecamylamine on cognition and behavior. Neuropsychopharmacology 10: 93-107.

Newhouse PA, Potter A, Kelton M, Corwin J (2001). Nicotinic treatment of Alzheimer's disease. Biol Psychiatry 49: 269-278.

Newhouse PA, Potter A, Lenox R (1993). The effects of nicotinic agents on human cognition: possible therapeutic applications in Alzheimer's and Parkinson's diseases. Medl Chem Res 2: 628-642.

Newhouse PA, Sunderland T, Tariot PN, Weingartner $\mathrm{H}$, Thomason K, Mellow AM et al (1988). The effects of acute scopolamine in geriatric depression. Arch Gen Psychiatry 45: 906-912.

Overall J, Gorham D (1993). The brief psychiatric rating scale. Psychol Rep 10: 799-812.

Packard MG (1998). Posttraining estrogen and memory modulation. Hormones Behav 34: 126-139.

Paganini-Hill A, Henderson VW (1994). Estrogen deficiency and risk of Alzheimer's disease in women. Am J Epidemiol 140: $256-261$.

Panidis DK, Matalliotakis IM, Rousso DH, Kourtis AI, Koumantakis EE (2001). The role of estrogen replacement therapy in Alzheimer's disease. Eur J Obstetr Gynecol Reprod Biol 95: 86-91.

Peterson R (1977). Scopolamine-induced learning failures in man. Psychopharmacology 52: 283-289.
Phillips SM, Sherwin BB (1992). Effects of estrogen on memory function in surgically menopausal women. Psychoneuroendocrinology 17: 485-495.

Polo-Kantola P, Portin R, Polo O, Helenius H, Irjala K, Erkkola R (1998). The effect of short-term estrogen replacement therapy on cognition: a randomized, double-blind, cross-over trial in postmenopausal women. Obstetr Gynecol 91: 459-466.

Rapp SR, Espeland MA, Shumaker SA, Henderson VW, Brunner $\mathrm{RL}$, Manson JE et al (2003). Effect of estrogen plus progestin on global cognitive function in postmenopausal women. J Am Med Assoc 289: 2663-2672.

Reisberg B, Ferris SH, de Leon MJ, Crook T (1988). Global detrioration scale (GDS). Psychopharmacol Bull 24: 661-663.

Resnick SM, Metter EJ, Zonderman AB (1997). Estrogen replacement therapy and longitudinal decline in visual memory: a possible protective effect. Neurology 49: 1491-1497.

Salthouse T (1996). The processing-speed theory of adult age differences in cognition. Psychol Rev 103: 401-428.

Sarter M, Bruno JP (2004). Developmental origins of the agerelated decline in cortical cholinergic function and associated cognitive abilities. Neurobiol Aging 25: 1127-1139.

Sarter M, Bruno JP, Givens B (2003). Attentional functions of cortical cholinergic inputs: what does it mean for learning and memory? Neurobiol Learn Memory 80: 245-256.

Sarter M, Hasselmo ME, Bruno JP, Givens B (2005). Unraveling the attentional functions of cortical cholinergic inputs: interactions between signal-driven and cognitive modulation of signal detection. Brain Res Rev 48: 98-111.

Sherwin BB (1988). Estrogen and/or androgen replacement therapy and cognitive functioning in surgically menopausal women. Psychoneuroendocrinology 13: 345-357.

Shumaker SA, Legault C, Kuller L, Rapp SR, Thal L, Lane DS et al (2004). Conjugated equine estrogens and incidence of probable dementia and mild cognitive impairment in postmenopausal women. J Am Med Assoc 291: 2947-2957.

Shumaker SA, Legault C, Rapp SR, Thal L, Wallace RB, Ockene JK et al (2003). Estrogen plus progestin and the incidence of dementia and mild cognitive impairment in postmenopausal women. J Am Med Assoc 289: 2651-2662.

Simpkins JW, Green PS, Gridley KE, Singh M, de Fiebre NC, Rajakumar G (1997). Role of estrogen replacement therapy in memory enhancement and the prevention of neuronal loss associated with Alzheimer's disease. Am J Med 103: 19S-25S.

Singh M, Meyer EM, Huang FS, Millard WJ, Simpkins JW (1993). Ovariectomy reduced ChAT activity and NGF mRNA levels in the frontal cortex and hippocampus of the female SpragueDawley rat. Soc Neurosci Abstr 19: 514.11 .

Sitaram N, Weingartner H, Gillin JC (1978). Human serial learning: enhancement with arecoline and choline and impairment with scopolamine. Science 201: 274-276.

Smith YR, Giordani B, Lajiness-O'Neill R, Zubieta J (2001a). Longterm estrogen replacement is associated with improved nonverbal memory and attentional measures in postmenopausal women. Fertil Steril 76: 1101-1107.

Smith YR, Minoshima S, Kuhl DE, Zubieta JK (2001b). Effects of long-term hormone therapy on cholinergic synaptic concentrations in healthy postmenopausal women. J Clin Endocrinol Metab 86: 679-684.

Sohrabji F, Green LA, Miranda R, Toran-Allerand CD (1994a). Reciprocal regulation of estrogen and nerve growth factor receptors by their ligands in PC12 cells. J Neurobiol 14: 974-988.

Sohrabji F, Miranda RC, Toran-Allerand CD (1994b). Estrogen differentially regulates estrogen and nerve growth factor receptor mRNAs in adult sensory neurons. I Neurosci 14: $459-471$.

Sunderland T, Tariot P, Murphy DL, Weingartner H, Mueller EA, Cohen RM (1985). Scopolamine challenges in Alzheimer's disease. Psychopharmacology 87: 247-249. 
Sunderland T, Tariot PN, Newhouse PA (1988). Differential responsivity of mood, behavior, and cognition to cholinergic agents in Alzheimer's disease. Brain Res Rev 13: 371-389.

Tang MX, Jacobs D, Stern Y, Marder K, Schofield P, Gurland B et al (1996). Effect of estrogen during menopause on risk and age at onset of Alzheimer's disease. Lancet 348: 429-432.

Tinkler GP, Tobin JR, Voytko ML (2004). Effects of two years of estrogen loss or replacement on nucleus basalis cholinergic neurons and cholinergic fibers to the dorsolateral prefrontal and inferior parietal cortex of monkeys. J Comp Neurol 469: 507-521.

Tinkler GP, Voytko ML (2005). Estrogen modulates cognitive and cholinergic processes in surgically menopausal monkeys. Prog Neuro-Psychopharmacol Biol Psychiatry 29: 423-431.

Toran-Allerand CD (1996). The estrogen/neurotrophin connection during neural development: is co-localization of estrogen receptors with the neurotrophins and their receptors biologically relevant? Dev Neurosci 18: 36-48.

van Amelsvoort TAMJ, Abel KM, Robertson DMR, Daly E, Critchley H, Whitehead $M$ et al (2001). Prolactin response to d-fenfluramine in postmenopausal women on and off ERT: comparison with young women. Psychoneuroendocrinology 26: 493-502.

Verghese J, Kuslansky G, Katz MJ, Sliwinski M, Crystal HA, Buschke $\mathrm{H}$ et al (2000). Cognitive performance in surgically menopausal women on estrogen. Neurology 55: 872-874.
Verhaeghen P, Salthouse T (1997). Meta-analysis of age-cognition relations in adulthood: estimates of linear and nonlinear age effects and structural models. Psychol Bull 122: 231-249.

Vermeer SE, Prins ND, den Heijer T, Hofman A, Koudstaal PJ, Breteler MMB (2003). Silent brain infarcts and the risk of dementia and cognitive decline. $N$ Engl J Med 348: 1215-1222.

Voytko ML (2002). Estrogen and the cholinergic system modulate visuospatial attention in monkeys (Macaca fascicularis). Behav Neurosci 116: 187-197.

Voytko ML, Tinkler GP (2004). Cognitive function and it's neural mechanisms in nonhuman primate models of aging, Alzheimer's Disease, and menopause. Front Biosci 9: 1899-1914.

Wechsler D (1981). Wechsler Adult Intelligence Scale-Revised. The Psychological Corporation: San Antonio.

Wechsler D (1997). Wechsler Memory Scale-Revised. The Psychological Corporation: San Antonio.

Woolley CS, McEwen BS (1993). Roles of estradiol and progesterone in regulation of hippocampal dendritic spine density during the estrous cycle in the rat. J Comp Neurol 336: 293-306.

Yaffe K (2003). Hormone therapy and the brain: déjà vu all over again? J Am Med Assoc 289: 2717-2719.

Zandi PP, Carlson MC, Plassman BL, Welsh-Bohmer KA, Mayer LS, Steffens DC et al (2002). Hormone replacement therapy and incidence of Alzheimer disease in older women. J Am Med Assoc 288: 2123-2129.

Supplementary Information accompanies the paper on Neuropsychopharmacology website (http://www.nature.com/npp) 